\title{
Environmentally-Friendly Magnetoelectric Ceramic Multilayer Composites by Water-based Tape Casting
}

\author{
Washington Santa-Rosa ${ }^{\mathrm{a}}$, Michel Venet ${ }^{\mathrm{a}}$, Jean-Claude M'Peko ${ }^{\mathrm{b}}$, Rodrigo \\ Moreno $^{c}$, Harvey Amorín ${ }^{\mathrm{d}}$, Miguel Algueród,* \\ ${ }^{a}$ Departamento de Física, Universidade Federal de São Carlos, 13565-905, São Carlos, \\ SP, Brazil. \\ ${ }^{b}$ Instituto de Física de São Carlos, Universidade de São Paulo, 13560-970, São Carlos, \\ $S P$, Brazil. \\ ${ }^{c}$ Instituto de Cerámica y Vidrio (ICV), CSIC. Cantoblanco, 28049 Madrid, Spain \\ ${ }^{d}$ Instituto de Ciencia de Materiales de Madrid, CSIC. Cantoblanco, 28049 Madrid, \\ Spain.
}

\section{Abstract}

Magnetoelectric composites are an enabling material technology for a range of novel devices like electrically-tunable magnetic microwave components or room-temperature-operation high-sensitivity magnetic sensors. Among the different approaches under development, cofired ceramic layered composites provide large effective magnetoelectric coefficients and improved reliability. However, miniaturization and processing up-scaling remain an issue. This can be addressed by using tape casting technology to prepare multilayer structures, as it is industrially done for multilayer ceramic capacitors. We report here the processing of ceramic multilayer composites of environmentally-friendly piezoelectric $\left(\mathrm{K}_{0.5} N a_{0.5}\right)_{0.96} \mathrm{Li}_{0.04} \mathrm{Nb} b_{1-y} \mathrm{Ta}_{y} \mathrm{O}_{3}$ and magnetostrictive $\mathrm{CoFe}_{1.75} \mathrm{Mn}_{0.25} \mathrm{O}_{4}$ by water-based tape casting. Dense ceramic multilayers with high quality interfaces were obtained, and their func-

\footnotetext{
${ }^{*}$ Corresponding author

Email address: malguero@icmm.csic.es (Miguel Algueró)
} 
tional response characterized. Effects of the multilayer geometry, characterized by ceramic layer thickness of tens of microns and large number of layers, have been defined. A distinctive enhancement of functionality as compared with conventionally-prepared layered structures is demonstrated and related to strain/stress relaxation characteristics across layers.

Keywords: Magnetoelectric materials, Layered composites, Tape casting, Environmentally-friendly technologies, Geometry-property relationships

\section{Introduction}

Magnetoelectric materials develop a linear electrical polarization in response to an applied magnetic field (direct effect), and a magnetization in response to an electric field (converse effect)[1]. A main line of research is single-phase multiferroics[2], and advances have recently been made towards obtaining compounds with room-temperature coupled electrical and magnetic ferroic orderings and distinctive magnetoelectric response[3, 4]. Alternatively, magnetoelectric coupling can be achieved by composite approaches. Rationale is to combine ferroelectric and ferromagnetic phases, so that magnetoelectricity is obtained from their interaction, either electrostatic, magnetic or elastic $[5,6]$. A range of potentially disruptive magnetoelectric thin film and bulk technologies have been proposed[7-11], among which magnetic sensing stands out as the closest to the market[12, 13].

The largest effective room-temperature magnetoelectric coefficients have been obtained for composites consisting of high-sensitivity piezoelectric and magnetostrictive phases, for which magnetoelectricity is obtained as a product property of their respective piezoresponses[14, 15]. Different piezorespon- 
sive phases and connectivity schemes are under consideration, but mostly ferroelectric oxides or polymers have been used as piezoelectric component, while magnetic metal alloys or oxides are the choice for the magnetostrictive one[16-18]. All 0-3, 1-3 and 2-2 composite configurations are being extensively explored for different applications[19-21]. In this notation, the first and second digits stand for the connectivity of the magnetic and dielectric phases, respectively. For instance, 0-3 refers to magnetostrictive particles embedded in a piezoelectric matrix, and 2-2 to stacks of magnetostrictive and piezoelectric layers.

Among bulk composite materials, cofired ceramic layered structures concentrate an increasing attention, because they offer improved reliability in applications and are more adequate for mass production and miniaturization[22]. Most of the work has been carried out on two- and three-layer composite structures obtained by conventional uniaxial pressing techniques, where powders are sequentially poured into a mould, and subsequently pressed to form a green body. A sintered ceramic body is obtained after a firing step. High quality composites with very high magnetoelectric coefficients have been obtained by using state-of-the-art $\mathrm{Pb}(\mathrm{Zr}, \mathrm{Ti}) \mathrm{O}_{3}$ (PZT) perovskite oxide and the $\mathrm{NiFe}_{2} \mathrm{O}_{4}$ spinel oxide[23, 24]. However, activity is currently focused on replacing PZT by a lead-free alternative after recently enforced environmental regulations like EU-Directive 2002/95/EC (RoHS) that requires the restriction of the use of lead, among a list of hazardous substances, in electrical and electronic equipment. Most promising alternatives are modifications of $(\mathrm{K}, \mathrm{Na}) \mathrm{NbO}_{3}(\mathrm{KNN})[25,26]$, and indeed layered composites of KNN-based compositions and magnetic spinel oxides have recently been reported with 
magnetoelectric performance comparable to that of PZT-based ones[27, 28]. Note that substitution of $\mathrm{NiFe}_{2} \mathrm{O}_{4}$ by a nickel-free alternative magnetostrictive oxide should also be carried out, if an utter environmentally-friendly material technology is targeted[29].

A major issue relating these two- and three-layer composite structures is miniaturization, since individual layer thickness is typically of several hundreds of microns. Besides, the processing is hardly up-scalable. Miniaturization and scalability can be addressed by using tape casting technologies to process multilayer structures, just like multilayer ceramic capacitors and actuators are industrially prepared $[30,31]$. However, attempts to apply this approach to magnetoelectric composites have been scarce, and contradictory results were obtained concerning the effects of the new geometry characterized by ceramic layer thickness of tens of microns and large number of layers $[23,32-34]$.

We report here the processing and functional characterization of high quality ceramic multilayer structures of KNN-based materials and a modified $\mathrm{CoFe}_{2} \mathrm{O}_{4}$ spinel oxide. A water-based tape casting formulation was developed to obtain an all-around environmentally-friendly material technology, which comply with current regulations. Performance was compared with that of ceramic three-layer structures with varying geometry, which enabled a comprehensive discussion of the effects of the multilayer configuration. Indeed, a distinctive enhancement of magnetoelectric response, directly related to the strain field relaxation characteristics, is clearly demonstrated. 


\section{Material and methods}

Powders of $\left(K_{0.5} N a_{0.5}\right)_{0.96} L i_{0.04} N b_{1-y} T a_{y} O_{3}$ with y=0.15 (KNLNT15) and $\mathrm{y}=0.20$ (KNLNT20) were synthesized by solid state reaction of stoichiometric mixtures of $\mathrm{K}_{2} \mathrm{CO}_{3}$ (Aldrich, > $99 \%$ pure), $\mathrm{Na}_{2} \mathrm{CO}_{3}$ (Aldrich, $>99 \%$ pure), $\mathrm{Li}_{2} \mathrm{CO}_{3}$ (Alfa Aesar, > $99.999 \%$ pure), $\mathrm{Nb}_{2} \mathrm{O}_{5}$ (CBMM, > $99.5 \%$ pure) and $\mathrm{Ta}_{2} \mathrm{O}_{5}$ (Across, $>99.99 \%$ pure). Two successive thermal treatments at $750{ }^{\circ} \mathrm{C}$ and $800{ }^{\circ} \mathrm{C}$ for $5 \mathrm{~h}$ were carried out, with an intermediate ball milling for $24 \mathrm{~h}$ in isopropyl alcohol with $\mathrm{Y}_{2} \mathrm{O}_{3}$-stabilized zirconia grinding media to increase chemical homogeneity[29]. KNLNT20 is a slight modification of $\left(K_{0.5} N a_{0.5}\right)_{0.97} L i_{0.03} N b_{0.8} \mathrm{Ta}_{0.2} \mathrm{O}_{3}$, named LF3 in Ref. 25, and that was highlighted as a viable alternative to replace PZT based materials. It was selected after a previous study that explored compositions around LF3, and that showed KNLNT20 to present enhanced piezoelectric coefficients[35]. In the same work, $\mathrm{KNLNT15/CoFe} \mathrm{O}_{2}$ composites were

anticipated to present the highest $\alpha_{31}^{E}$ among a range of $\mathrm{KNLNT} / \mathrm{CoFe}_{2} \mathrm{O}_{4}$ layered structures.

$\mathrm{CoFe}_{1.75} \mathrm{Mn}_{0.25} \mathrm{O}_{4}$ (CFM25) was used as magnetostrictive component. Mn substitution for Fe has been demonstrated to be an effective means of reducing the magnetocrystalline anisotropy of $\mathrm{CoFe}_{2} \mathrm{O}_{4}$, and to provide the enhanced rate of change of magnetostriction with applied magnetic field required for its use in magnetoelectric composites[36]. This is necessary because the high magnetocrystalline anisotropy and coercive field of $\mathrm{CoFe}_{2} \mathrm{O}_{4}$ as compared with $\mathrm{NiFe}_{2} \mathrm{O}_{4}$ results in a reduced rate of change of magnetostriction and thus, of effective piezomagnetic coefficients under bias, even if the total magnetostriction of $\mathrm{CoFe}_{2} \mathrm{O}_{4}$ is larger than that of $\mathrm{NiFe}_{2} \mathrm{O}_{4}$. Highly reactive 
CFM25 powders were prepared by the Pechini method. In this procedure, stoichiometric amounts of the starting materials $\mathrm{Fe}\left(\mathrm{NO}_{3}\right)_{3}, \mathrm{Co}\left(\mathrm{NO}_{3}\right)_{2}$ and $\mathrm{Mn}\left(\mathrm{NO}_{3}\right)_{2}$ (Across, >99\% pure) were dissolved in an aqueous citric acid solution. Acid in 3:1 M ratio with respect to metal nitrates was used. Subsequently, ethylene glycol was added in 2:3 ratio with respect to the citric acid. The sol was then heated at $90^{\circ} \mathrm{C}$ until its complete transformation into a gel. Finally, the obtained gel was dried at $300{ }^{\circ} \mathrm{C}$ for $4 \mathrm{~h}$, and thermally treated at $600{ }^{\circ} \mathrm{C}$ for $1 \mathrm{~h}$ to obtain the spinel oxide. This procedure has been recently applied to the preparation of highly reactive $\mathrm{CoFe}_{2} \mathrm{O}_{4}(\mathrm{CFO})$ and $\mathrm{CoFe}_{1.85} \mathrm{Mn}_{0.15} \mathrm{O}_{4}$ (CFG) powders, which enabled the low temperature processing of $\mathrm{CFO} / \mathrm{KNN}$ and $\mathrm{CFG} / \mathrm{KNLNT}$ layered composites[29].

Water-based tape casting formulations were developed for both the KNLNT and CFM powders. Stable powder suspensions in deionised water were obtained by using the cationic polymeric dispersant Hypermer KD2 up to 2 wt\% on a dry solid basis, and a Dr. Hielscher UP400S high energy ultrasonic probe. Best results were obtained with initial solid loadings of 35 and 22.5 vol\% for KNLNT and CFM, respectively, and 2 minutes dispersing time. DURAMAX B-1000 acrylic emulsion binder was then added to the suspensions to a total content of $10 \mathrm{wt} \%$ on a dry solid basis to obtain the final slurries for tape casting. This emulsion contains $55 \%$ active matter. Details of the rheological studies that lead to the optimization of suspensions and slurries will be reported separately.

Slurries were cast with a single blade system, the blade height fixed at $200 \mu \mathrm{m}$ and a blade speed of $30 \mathrm{~mm} \cdot \mathrm{s}^{-1}$ (so that the calculated shear rate operating through the blade is $150 \mathrm{~s}^{-1}$ ) on silicone-coated Mylar substrates. 
KNLNT and CFM tapes with thickness $\sim 175$ and $\sim 135 \mu \mathrm{m}$, respectively, were obtained after drying. Tapes were then alternatively stacked and laminated under an uniaxial pressure of $30 \mathrm{MPa}$. Green ceramic nine-layer structures, consisting of four CFM/KNLNT bilayers plus an additional end CFM layer, were obtained after organics were burned out at $500{ }^{\circ} \mathrm{C} / 1 \mathrm{~h}$, with slow heating/cooling rates of $1{ }^{\circ} \mathrm{C} \cdot \mathrm{min}^{-1}$ to avoid delamination.

Finally, ceramic multilayer structures with targeted piezoelectric volumetric fraction $r_{p} \sim 0.33$ were processed by hot pressing of the green stacks at 1050 ${ }^{\circ} \mathrm{C}$ under $60 \mathrm{MPa}$, using an Alfred Ceramic Enterprises, INC press. Heating/cooling rates of $+3 /-1^{\circ} \mathrm{C} \cdot \mathrm{min}^{-1}$, and a soaking time of $1 \mathrm{~h}$ were selected. Pressure was applied once the maximum temperature was reached, and maintained during soaking and cooling down to room-temperature. These conditions have previously been used for the processing of high quality ceramic three-layer structures of CFG and KNLNT[29].

Cross section samples perpendicular to the stacking plane were prepared for microstructural characterization by polishing with $\mathrm{Al}_{2} \mathrm{O}_{3}$ suspensions down to $0.1 \mu \mathrm{m}$. Composite interface quality and ceramic microstructures across the sample were characterized by scanning electron microscopy with a FEI (Inspect F-50) apparatus. Possible interdiffusion phenomena across interfaces were investigated by energy dispersive X-ray spectroscopy (EDXS) in the same microscope, equipped with an Apollo X Silicon Drift Detector. The occurrence of chemical reactions at the interface was further studied with micro-Raman spectroscopy. A WITec alpha300 R confocal Raman imaging system with $514 \mathrm{~nm}$ wavelength excitation was used. A 100x objective and a numerical aperture of $0.82(\mathrm{NA})$ were selected, which resulted in a light spot 
of $\sim 0.4 \mu \mathrm{m}$ diameter on focus.

Ceramic capacitors for the electrical characterization of the composite multilayer structures were prepared by painting $\mathrm{Ag}$ electrodes on the major faces, and subsequent sintering at $700{ }^{\circ} \mathrm{C}$. The temperature dependences of the dielectric permittivity and losses were measured between room-temperature (RT) and $550{ }^{\circ} \mathrm{C}$ with a Hewlett Packard HP4284A precision LCR meter. Measurements were dynamically carried out during a heating/cooling cycle with $\pm 1.5^{\circ}$ C. min $^{-1}$ rate at several frequencies between $100 \mathrm{~Hz}$ and $1 \mathrm{MHz}$. RT ferroelectric hysteresis loops were recorded under voltage sine waves of increasing amplitude up to $10 \mathrm{kV}$ with frequencies between 1 and $10 \mathrm{~Hz}$, obtained by the combination of a HP 3325B synthesizer/function generator and a TREK 10/40 high-voltage amplifier, while charge was measured with a homebuilt charge to voltage converter and software for loop acquisition and analysis.

Subsequently, the composite multilayer structures were poled for piezoelectric characterization. Optimum conditions were defined by applying increasing fields at temperatures between RT and $100{ }^{\circ} \mathrm{C}$, until saturation of the piezoelectric charge longitudinal coefficient $d_{33}$ was attained as measured with a Berlincourt type meter $24 \mathrm{~h}$ after the poling step, or dielectric breakdown occurred.

Finally, the magnetoelectric response of the composite layered structures was characterized. A system comprising a combination of one electromagnet and one Helmholtz coil, designed to independently provide a static magnetic field up to $10 \mathrm{kOe}$ to magnetize the material, and an alternate magnetic field of $30 \mathrm{Oe}$ at $25 \mathrm{~Hz}$ that plays as stimulus was used, while the magnetoelectric 
voltage response was monitored with a lock-in amplifier. A 3-1 geometry was chosen to obtain the transverse magnetoelectric coefficient $\alpha_{31}^{E}$ as a function of the bias magnetic field $\mathrm{H}$, after normalization to the piezoelectric element thickness.

To support discussion, a number of three-layer composite structures with large single layer thickness between 0.5 and $1 \mathrm{~mm}$, and $r_{p}$ of either 0.33 or 0.5 were also processed. Piezoelectric and magnetostrictive oxide powders were initially shaped in three-layer stacks by uniaxial pressing, and subsequently hot pressed with the same conditions used for the multilayered structures.

Both CFM/KNLNT/CFM and KNLNT/CFM/KNLNT configurations were prepared and characterized.

\section{Results}

Dense ceramic multilayer composites with very good bonding between layers were obtained as it is illustrated in Fig. 1a, where a scanning electron microscopy image of a CFM15/KNLNT20 nine-layer structure is shown. Final magnetostrictive and piezoelectric single layer thickness of $\sim 60$ and $\sim 40$ $\mu \mathrm{m}$ resulted, respectively. This corresponds to an actual piezoelectric volume fraction $r_{p}$ of 0.35 , which allows a direct comparison with the three-layer structures.

High-magnification images of an interface (Fig. 1b) and of the ceramic microstructures of the magnetostrictive (1c) and piezoelectric (1d) layers are also given. It is remarkable the high quality of the interfaces, which are free of microstructural defects. Microstructural gradients are not found within layers, and a similar average grain size of the order of the micron is found 

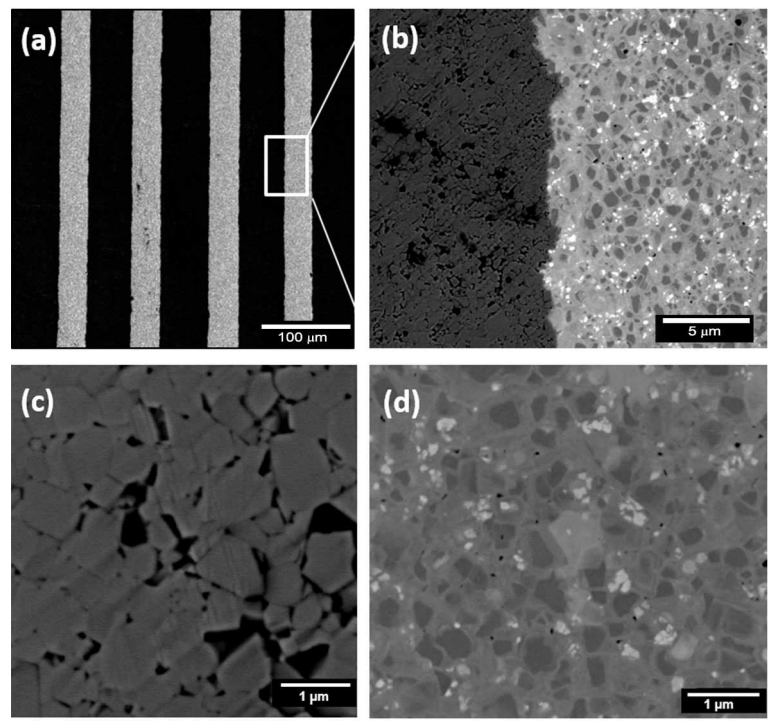

Figure 1: SEM images of a CFM25/KNLNT20 multilayer structure: (a) overall view, (b) interface, (c) ceramic microstructure of the CFM25 component, and (d) of the KNLNT20 component.

for the KNLNT and CFM layers, while densification was slightly higher for the piezoelectric component.

Chemical line scans and mappings obtained by EDXS across interfaces are provided in Fig. 2 and the supplementary information (Fig. S1), respectively. No indication of interdiffusion was found, at least within the sensitivity of the technique.

Similarly, no evidence of local chemical reactions at interfaces that result in formation of second phases was met with micro-Raman. Spectra at four points across one interface are shown in Fig. 3. Note that analogous spectra are obtained at locations 1 (within the CFM layer $\sim 5 \mu \mathrm{m}$ away from the interface) and 2 (still in the CFM layer but next to the interface), while identical spectra are also obtained at locations 3 (already in the KNLNT 

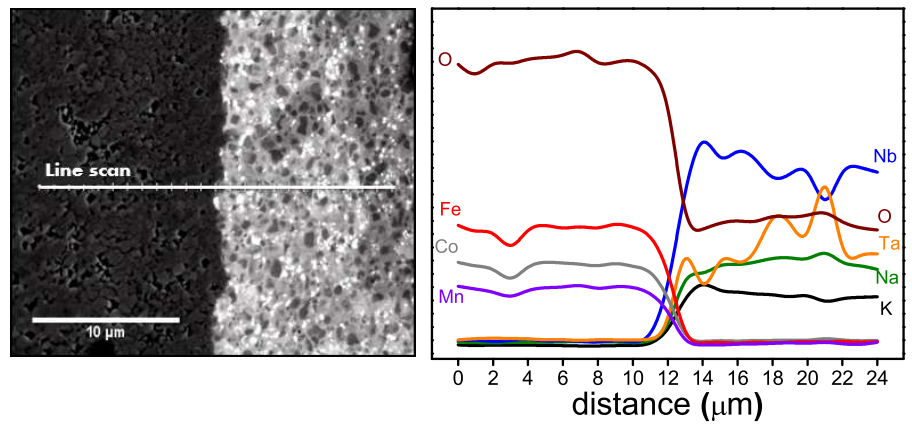

Figure 2: Chemical line scans across the CFM25/KNLNT20 multilayer interface, obtained by EDXS.

layer but next to the interface) and 4 (within the KNLNT layer phase $\sim 5$ $\mu \mathrm{m}$ away). No Raman bands other than those characteristic of CFM are found in 1 and 2, while no bands other than those characteristic of KNLNT are found in 3 and 4 . This indicates the absence of phase mixing or of second phases. The sharpness of the interface is well illustrated by the evolution of the integrated intensity of the $844-879 \mathrm{~cm}^{-1}$ Raman band shown in Fig. $3 c$, which corresponds to the $A_{1 g}+F_{2 g}$ modes of KNLNT. Note that the normalized integrated intensity goes from 0 to 1 in a single step. Lateral resolution is defined by the size of the laser spot focused on the sample that is below $0.5 \mu \mathrm{m}$. This value is already smaller than grain size. Overall, the microstructural, chemical and structural characterizations clearly reveal the high quality of interfaces in the multilayer ceramic composite. This is essential for having good mechanical coupling between the two phases and thus, for high magnetoelectric response.

The ceramic microstructure of the KNLNT layers deserves some comment. Individual grains show a distinctive core-shell structure, as illustrated in Fig. 1d. Core-shell structured grains with an average size of $\sim 0.6 \mu \mathrm{m}$ 

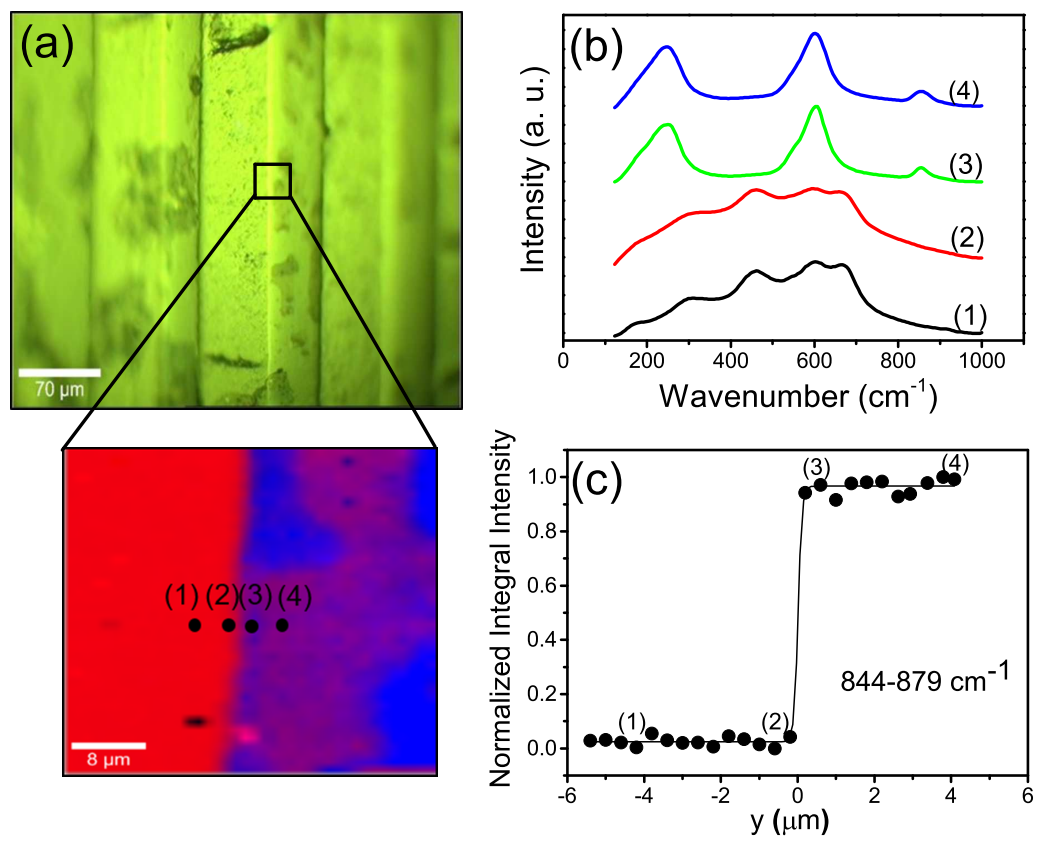

Figure 3: Micro-Raman studies across a CFM25/KNLNT20 interface: (a) optical image of the area indicating locations, at which (b) spectra were taken, (c) evolution of the integrated intensity of the $844-879 \mathrm{~cm}^{-1}$ Raman band corresponding to the $A_{1 g}+F_{2 g}$ modes of KNLNT. 
were present all across the piezoelectric layer, along with smaller, very bright grains. This microstructure is characteristic of samples prepared by direct mixing of alkaline carbonates and $\mathrm{Ta}$ and $\mathrm{Nb}$ oxides, and has been related to A- and B-cation concentration inhomogeneities, so that Ta- and Na-rich shells surrounded $\mathrm{Nb}$ - and $\mathrm{K}$ - rich cores, while smaller particles were Tarich. Chemical inhomogeneity was reduced by prolonged high temperature annealing, yet it could not be completely eliminated[29, 37]. Besides, alkali volatilization was triggered at high temperature, along with chemical reactions at piezoelectric/magnetostrictive interfaces and interdiffusion across them. A trade-off thus must be reached for maximizing the magnetoelectric response.

The high quality of the composite multilayer structures enabled a sound electrical characterization. Results are shown in Figs. 4, 5, 6, S2 and S3, where the properties of the multilayer composites are compared with those of conventionally-prepared three-layer structures with varying geometry. The temperature dependence of the relative permittivity is shown in Figs. 4 and S2. The characteristic dielectric anomaly associated with the ferroelectric transition is easily identified in the response of all the composite layered structures at a temperature that decreases when the amount of $\mathrm{Ta}$ substituting for $\mathrm{Nb}$ increases[38], so as it is at $\sim 375{ }^{\circ} \mathrm{C}$ and $\sim 345^{\circ} \mathrm{C}$ for KNLNT15- and KNLNT20-based materials, respectively. Permittivity also reflects chemical inhomogeneity as a distinctive high temperature shoulder that can be observed above the ferroelectric transition temperature for all multilayer and three-layer structures (marked with an arrow in Fig 4a). This additional anomaly takes place at $\sim 420^{\circ} \mathrm{C}$, which is the temperature of the 
ferroelectric transition for $\mathrm{K}_{0.5} \mathrm{Na}_{0.5} \mathrm{NbO}_{3}$. This indicates cores to be KNN surrounded by KNLNT shells, as confirmed by EDXS[29]. Note that permittivity increases with the piezoelectric volumetric fraction as expected, while the specific configuration, either multilayer or three-layer, and layer sequence, either CFM/KNLNT/CFM or KNLNT/CFM/KNLNT have no clear effect.

Frequency dispersion in permittivity is also presented in the Fig. 4. All composite layered structures showed a step-like increase of permittivity at intermediate temperatures, whose height decreased as frequency increased, while its positions shifted toward higher temperatures. Corresponding loss tangent values are shown in Figs. 5 and 3S. A distinctive peak in losses is associated with the highly dispersive step. This behavior has widely been observed in layered composites, and it is associated with a Maxwell-Wagner type relaxation originated from the difference in conductivity between the piezoelectric perovskite and magnetostrictive spinel oxides[29, 32]. Note that the step position shifts down to low temperature for the multilayer structure as compared with a three-layer structure with similar piezoelectric volumetric fraction. This is what one expects for a Maxwell-Wagner type relaxation when the single-layer thickness of the conductive component is reduced, as it is the case here. A decrease of approximately an order of magnitude, from $\sim 500 \mu \mathrm{m}$ in the three-layer structure to $\sim 50 \mu \mathrm{m}$ in the multilayer takes place.

Room-temperature ferroelectric hysteresis loops for the multilayer composite and for selected three-layer structures are shown in Fig. 6. Both CFM/KNLNT/CFM and KNLNT/CFM/KNLNT configurations are compared with the multilayer. Remnant polarization $P_{R}$ and coercive field $E_{C}$ values of $\sim 11 \mu \mathrm{C} . \mathrm{cm}^{-2}$ and $\sim 6 \mathrm{kV} . \mathrm{mm}^{-1}$ were found. Polarization is 


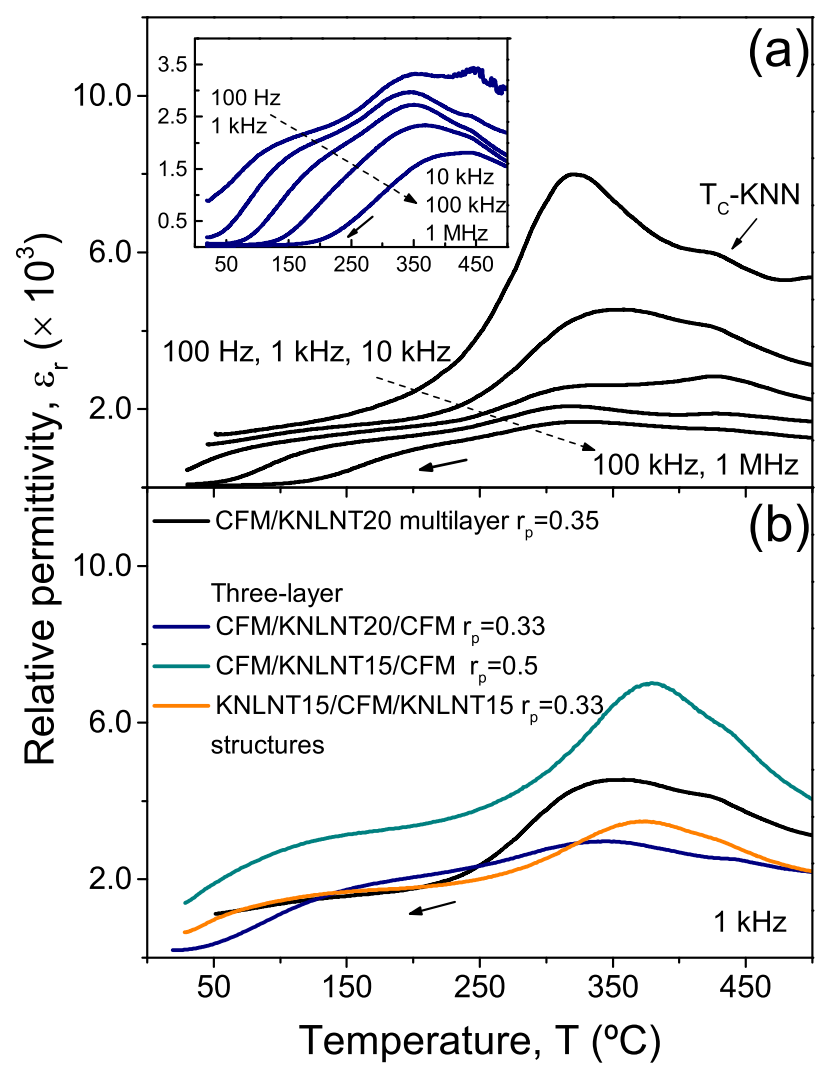

Figure 4: Temperature dependence of permittivity for (a) a CFM25/KNLNT20 multilayer structure with piezoelectric volumetric fraction $r_{p}=0.35$ at several frequencies, and (b) its comparison with the permittivity of three-layer structures with varying configuration and $r_{p}$. Inset in (a) shows permittivity at five frequencies for a CFM25/KNLNT20/CFM25 three-layer structure with $r_{p}=0.33$. 


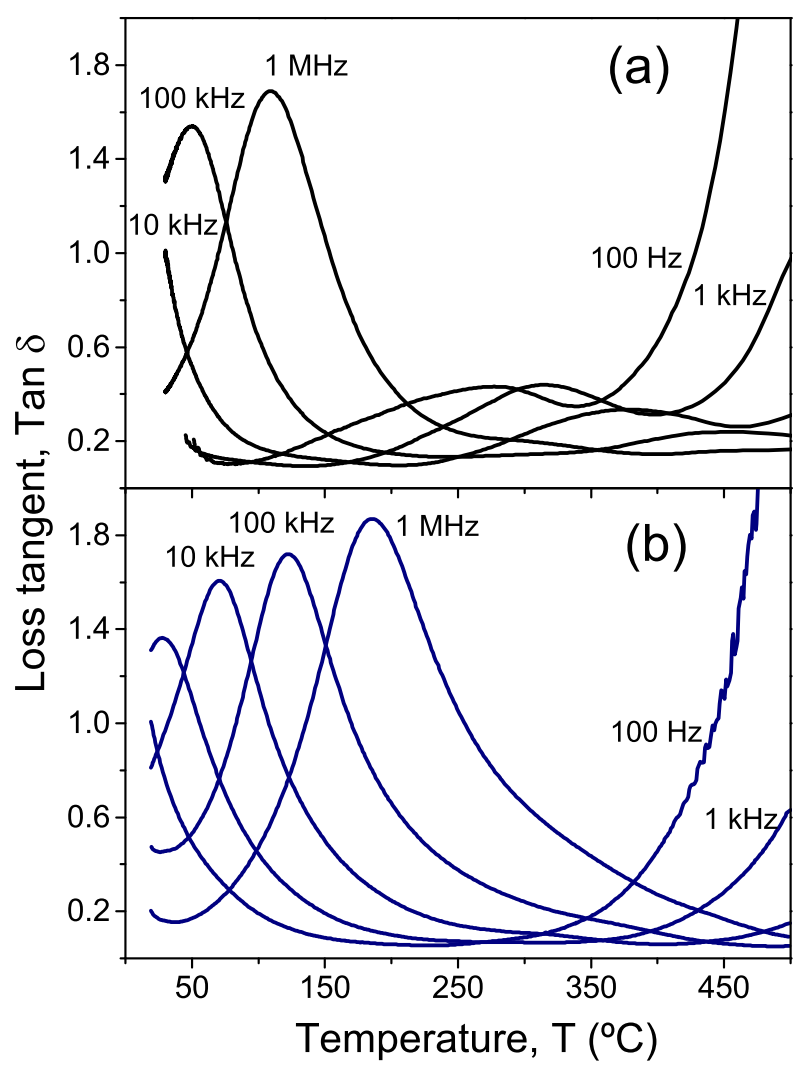

Figure 5: Temperature dependence of the loss tangent of (a) a CFM25/KNLNT20 multilayer structure with piezoelectric volumetric fraction $r_{p}=0.35$, and (b) a CFM25/KNLNT20/CFM25 three-layer structure with $r_{p}=0.33$, both at several frequencies. 
slightly lower than that for three-layer structures of $\sim 14 \mu \mathrm{C} . \mathrm{cm}^{-2}$. On the contrary, coercive field is well above the value of $\sim 2.5 \mathrm{kV} . \mathrm{mm}^{-1}$ obtained for conventionally-prepared layers. Actually, in the previous investigation of CFG/KNLNT/CFG layered composites[29], $E_{C}$ was shown to be significantly higher than that of KNLNT piezoelectric ceramics analogously processed, and effect that was related to the presence of the CFG two layers in series with the KNLNT one that have large capacitive impedances and thus, causes a decrease of the effective electric field across the piezoelectric. Number of interfaces in the multilayer structure significantly raises from 2 to 8 , so a further increase of effective coercivity would be expected, as observed. This also suggests that saturation might be increasingly difficult to achieve in multilayers, which would explain the lower $P_{R}$.

Slight differences between the three-layer structures might also be significant. Main one is a higher remnant polarization for the CFM/KNLNT/CFM structure than for the KNLNT/CFM/KNLNT one. In the mentioned previous work[29], CFG/KNLNT/CFG three-layer structures were found to present higher remnant polarization than KNLNT piezoelectric ceramics analogously processed. This effect was associated with the presence of compressive stresses within the piezoelectric layer. An enhancement of remnant polarization as a result of domain reorientation phenomena under substrate compressive stresses is a well known phenomenon in thin film technology[39]. Indeed CFM exerts in-plane compressive stress on the KNLNT layers, which one expects to be larger in the CFM/KNLNT/CFM configuration than in the KNLNT/CFM/KNLNT one. A higher remnant polarization can thus be anticipated in the former case. Relating coercive field, similar values were 


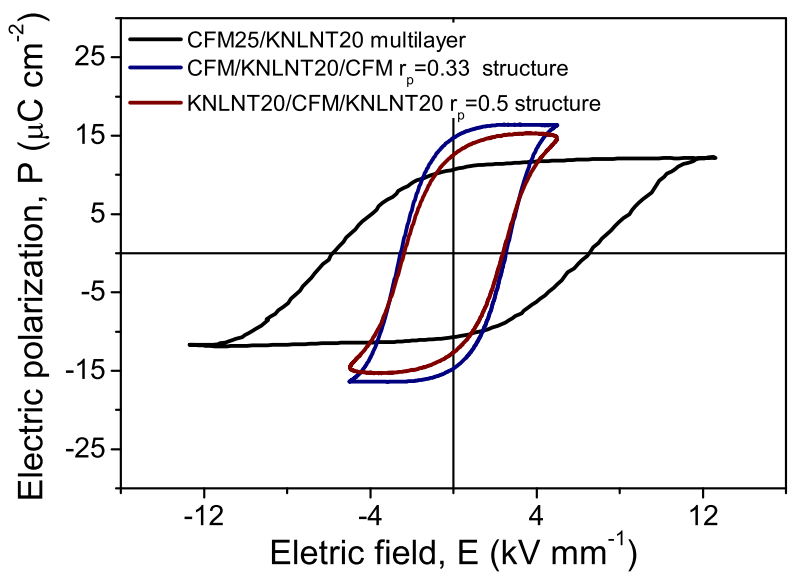

Figure 6: Ferroelectric hysteresis loop for a CFM25/KNLNT20 multilayer structure with piezoelectric volumetric fraction $r_{p}=0.35$, compared with those for three-layer structures with varying configuration and $r_{p}$.

obtained, consistently with the same number of interfaces.

Ceramic multilayer composites were poled with $8 \mathrm{kV} \cdot \mathrm{mm}^{-1}$ for $15 \mathrm{~min}$ at room temperature, which resulted in a $d_{33}$ piezoelectric coefficient of $\sim 35$ pC. $\mathrm{N}^{-1}$. Hot poling was not possible because of non-negligible conduction, and higher electric fields resulted in dielectric breakdown. This figure is within typical values of $30-45$ pC. $\mathrm{N}^{-1}$ for conventionally prepared composite three layer structures (with slightly larger values for the CFM/KNLNT/CFM configuration than for the KNLNT/CFM/KNLNT one). Comparison however is not straightforward, as dielectric strength was lower in this case, and poling fields exceeding $6 \mathrm{kV} \cdot \mathrm{mm}^{-1}$ could not be applied.

The bias magnetic field dependence of the transverse magnetoelectric coefficient $\alpha_{31}^{E}$ for the composite layered structures is shown in Fig. 7. Response was significantly larger: $\sim 30 \mathrm{mV} . \mathrm{cm}^{-1}$. Oe ${ }^{-1}$ for composite multilayer structures than for conventionally-prepared three-layer structures, whose coeffi- 
cients did not exceed $7 \mathrm{mV} \cdot \mathrm{cm}^{-1} \cdot \mathrm{Oe}^{-1}$. Besides, the bias field for maximum response that is $\geq 0.85 \mathrm{kOe}$ for the three-layer structures, shifts down to 0.5 kOe for the multilayer configuration. In Ref. 29, it was noted that microcracking within a CFO-based component under tensile in-plane stress caused a decrease of the maximum magnetoelectric coefficient, accompanied by a distinctive shift of the bias field for maximum response towards higher values. This bias field is characteristic of the magnetostrictive layer and independent of the mechanical coupling across the interface, unlike the magnetoelectric coefficient. Its shift then must be a consequence of the drastic tensile stress relaxation within this layer after microcracking, and it thus reflect the dependence of the magnetostriction characteristics on tensile stress. Basically this is an enhanced rate of change of magnetostriction with applied magnetic field under the stress field.

Indeed, results for the three-layer structures are consistent with such a stress effect. Note that the bias field for maximum response is distinctively lower for the KNLNT/CFM/KNLNT configuration: $\sim 0.85 \mathrm{kOe}$, than for the CFM/KNLNT/CFM one: 1.05-1.5 kOe (depending on piezoelectric volumetric fraction $r_{p}$ ). Larger tensile stresses are expected within the magnetostrictive layer when it is sandwiched between the piezoelectric layers than when it is placed outside sandwiching a piezoelectric one. Consistently, an enhanced rate of change of magnetostriction with applied magnetic field is obtained in the former case. Relating magnetoelectric coefficients, slightly larger magnetoelectric coefficients are obtained for the CFM/KNLNT/CFM configuration than for the KNLNT/CFM/KNLNT. This is also likely a stress effect but within the piezoelectric layer, for compressive stresses favor its poling and 


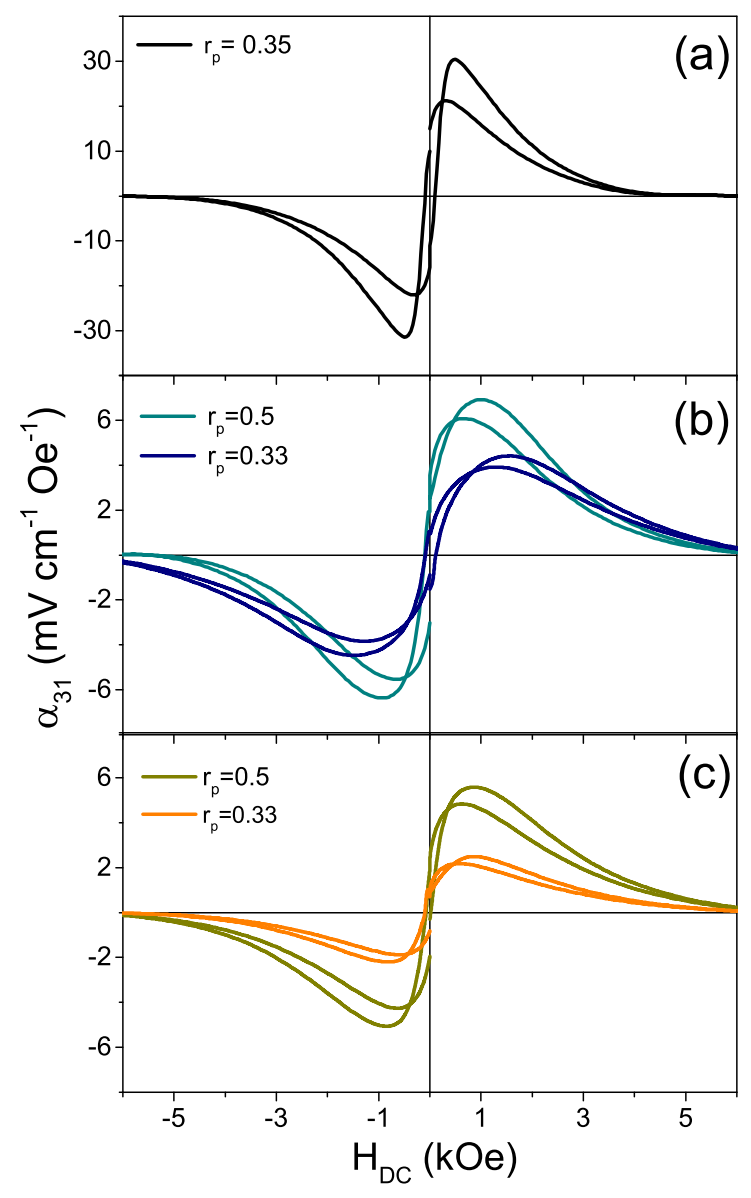

Figure 7: Bias magnetic field dependence of the transverse magnetoelectric coefficient for (a) a CFM25/KNLNT20 multilayer structure with piezoelectric volumetric fraction $r_{p}=0.35$, and (b) CFM25/KNLNT15/CFM25 and (c) KNLNT15/CFM25/KNLNT15 three-layer structures with varying $r_{p}$. The dashed line is a guide to the eye to highlight shifts in the bias field for maximum response. 
thus, result in larger piezoelectric coefficients. Poling level effects are also most probably behind the clear increase of magnetoelectric coefficients with piezoelectric volumetric fraction for both types of three-layer structures.

\section{Discussion}

Results here presented clearly indicate that dense ceramic multilayer composites with high quality interfaces have been obtained by using tape casting technology. They also show that the multilayer geometry, characterized by single-layer thickness of tens of microns and large number of layers, results in a higher magnetoelectric response than that of three-layer structures processed by conventional uniaxial pressing with typical single-layer thickness of hundreds of microns. Very similar results have previously been obtained for composite layered structures of $\mathrm{Bi}_{0.36} \mathrm{~Pb}_{0.64} \mathrm{Sc}_{0.36} \mathrm{Ti}_{0.64} \mathrm{O}_{3}$ and $\mathrm{NiFe}_{2} \mathrm{O}_{4}$. Coefficients of $36 \mathrm{mV} . \mathrm{cm}^{-1}$. Oe $\mathrm{e}^{-1}$ were obtained in that case for piezoelectric/magnetostrictive/piezoelectric three-layer structures, while a value of $108 \mathrm{mV} . \mathrm{cm}^{-1} \cdot \mathrm{Oe}^{-1}$ was obtained for a multilayer composite[32]. Note however that layered structures with different volumetric piezoelectric fraction $r_{p}$ of 0.66 and 0.4 , respectively, were prepared and studied in Ref. 32 .

Reports of composite layered structures of $\mathrm{Pb}(\mathrm{Zr}, \mathrm{Ti}) \mathrm{O}_{3}$ and magnetic spinel oxides can be also found, but they lead to contradictory results. A seminal work on ceramic multilayer structures of $\mathrm{Pb}(\mathrm{Zr}, \mathrm{Ti}) \mathrm{O}_{3}$ and $\mathrm{NiFe}_{2} \mathrm{O}_{4}$ showed magnetoelectric responses slightly smaller than ceramic two-layer structures of analogous piezoelectric phase fraction[23]. Note however that a distinctive increase of magnetoelectric coefficient was obtained for multilayers when the piezoelectric fraction $r_{p}$ was decreased by increasing the single-layer 
thickness of the magnetostrictive component. Specifically, a high magnetoelectric coefficient of $400 \mathrm{mV} \cdot \mathrm{cm}^{-1}$. Oe $\mathrm{e}^{-1}$ was reported in this work for a multilayer structure with $r_{p}=0.5$, which further raised up to $1.5 \mathrm{~V} . \mathrm{cm}^{-1} . \mathrm{Oe}^{-1}$ when the piezoelectric fraction was decreased down to $r_{p}=0.3125$, figure ever later matched. For instance, a value of $12 \mathrm{mV} \cdot \mathrm{cm}^{-1}$.Oe ${ }^{-1}$ was recently reported for multilayers of $\mathrm{Pb}_{0.99}\left[Z r_{0.45} \mathrm{Ti}_{0.47}\left(\mathrm{Ni}_{1 / 3} \mathrm{Sb}_{2 / 3}\right)_{0.08}\right] \mathrm{O}_{3}$ and $\mathrm{CoFe}_{2} \mathrm{O}_{4}$ with $r_{p}=0.78[33]$.

Indeed, comparatively low values of magnetoelectric coefficients are rather the rule than the exception, and specific studies to deepen into their causes have recently been accomplished using $\mathrm{Bi}_{0.36} \mathrm{~Pb}_{0.64} \mathrm{Sc}_{0.36} \mathrm{Ti}_{0.64} \mathrm{O}_{3} / \mathrm{NiFe}_{2} \mathrm{O}_{4}$ as a model system. $B i_{0.36} P b_{0.64} S c_{0.36} T i_{0.64} O_{3}$ is a high-Curie-temperature, high-sensitivity piezoelectric under consideration as an alternative to PZT for operation beyond $200{ }^{\circ} \mathrm{C}$ and up to $400{ }^{\circ} \mathrm{C}$ [40-42], and a response lower than those of PZT-based multilayers cannot be anticipated. A recent experimental study focused on studying local properties across interfaces, which were shown to be fully functional[43]. Low response therefore could not be associated with them. This fostered a simulation study by finite element analysis of possible mechanisms responsible of the reduction in response, which proposed strain relaxation across the piezoelectric layer as the most likely one[44]. A microstructure dependent characteristic relaxation length was found that ranged between 150 and $40 \mu \mathrm{m}$ for the specific material system. This explains why conventionally prepared three-layer structures show lower response than multilayer composites processed by tape casting technology. Basically, a reduction of response takes place when the piezoelectric singlelayer thickness is increased above the characteristic relaxation thickness. It 
is worth noting that piezoelectric single-layer thickness was of only $14 \mu \mathrm{m}$ in the case of the $\mathrm{PZT} / \mathrm{NiFe}_{2} \mathrm{O}_{4}$ multilayers of Ref. 23, and of $100 \mu \mathrm{m}$ in the case of the multilayers of Ref. 33. Intermediate values of 35 and $40 \mu \mathrm{m}$ were used in the $\mathrm{Bi}_{0.36} \mathrm{~Pb}_{0.64} \mathrm{Sc}_{0.36} \mathrm{Ti}_{0.64} \mathrm{O}_{3} / \mathrm{NiFe}_{2} \mathrm{O}_{4}$ and $\mathrm{KNLNT/CFM}$ multilayer structures of Ref. 32 and the present work, respectively.

Relaxation effects are also found in the bias magnetic field for maximum response, which directly relates to the stress state within the magnetostrictive layers. Tensile stresses develop within this layer during processing that affect magnetostriction by enhancing its rate of change with applied magnetic field. Relaxation phenomena like microcracking decrease the stress level and cause a reduction of rate and thus, a shift of the bias field for maximum response towards high values. This field was found to be significantly lower for the composite multilayer structures than for the three-layer ones. Stress relaxation thus is clearly minimized by the multilayer geometry. Note that low bias magnetic fields for maximum response are advantageous for applications. Therefore, a twofold positive effect of the multilayer geometry on functionality has been revealed, associated with the tailoring of strain/stress fields within the layered structures: (1) and enhancement of magnetoelectric coefficients as a result of the reduction of the piezoelectric single-layer thickness below the characteristic relaxation length, and (2) a shift of the bias magnetic field for maximum response towards low values as a result of unrelaxed tensile stresses within the magnetostrictive component. Note that relaxation behavior can be expected to differ among material systems, and to also strongly depend on microstructure. This is most probably behind contradictory results between multilayers with different single-layer thickness, 
phase volumetric fraction or even just processing. Nonetheless, and in the case of the KNLNT/CFM composite multilayer structures here processed and studied, multilayer geometry clearly resulted in enhanced functionality.

It must be noted that the magnetoelectric response of the KNLNT/CFM layered composites is limited by chemical inhomogeneity within the piezoelectric layers. Indeed, the role of the characteristic core-shell microstructure in degrading the piezoelectric performance of KNLNT piezoceramics has been previously discussed[37]. Optimized KNLNT compositions are those, for which the ferroelectric polymorphic phase transition between the tetragonal and orthorhombic phases has been shifted down to room temperature by $\mathrm{Ta}$ and $\mathrm{Li}$ substitutions. Local deviations from the average composition at both grain cores and shells, even if small, have a strong effect on the piezoelectric response and thus, on the magnetoelectric one of any composite structure containing them. Therefore, an enhancement of response can be expected if chemical homogeneity is improved at the powder level, always maintaining the high reactivity required for its co-sintering with CFM. In Ref. 37, pre-reaction of the $\mathrm{Nb}_{2} \mathrm{O}_{5}$ and $\mathrm{Ta}_{2} \mathrm{O}_{5}$ precursor oxides was demonstrated as an effective means of avoiding the formation of the core-shell grained microstructures and improving piezoelectric properties. Wet chemistry and mechanochemical activation techniques are also promising $[45,46]$. An alternative is to work with Ta-free KNN-based compositions, like $\left(K_{0.5} N a_{0.5}\right)_{1-x} L i_{x} O_{3}$. This system also present high piezoelectric coefficients[38], and $\alpha_{31}^{E}$ coefficients of $\sim 300 \mathrm{mV} \cdot \mathrm{cm}^{-1}$.Oe $\mathrm{e}^{-1}$ have been recently reported for composite $\left(\mathrm{K}_{0.48} \mathrm{Na}_{0.535}\right)_{0.942} \mathrm{Li}_{0.058} \mathrm{NbO}_{3} / \mathrm{Co}_{0.6} \mathrm{Zn}_{0.4} \mathrm{Fe}_{1.7} \mathrm{Mn}_{0.3} \mathrm{O}_{4}$ layered 
structures[28, 34].

\section{Summary and Conclusions}

Dense $\quad\left(K_{0.5} \mathrm{Na}_{0.5}\right)_{0.96} \mathrm{Li}_{0.04} \mathrm{Nb}_{1-y} \mathrm{Ta}_{y} \mathrm{O}_{3} / \mathrm{CoF} e_{0.75} \mathrm{Mn}_{0.25} \mathrm{O}_{4} \quad$ multilayer composites with very good bonding between layers and high quality interfaces can be obtained by water-based tape casting technology and hot pressing at a temperature as low as $1050{ }^{\circ} \mathrm{C}$. No indication of chemical reactions at interfaces or interdiffusion across them is found, while alkali volatilization is effectively suppressed at this temperature. A complete electrical, piezoelectric and magnetoelectric characterization has been carried out, and functionality compared with that of composite three-layer structures prepared by conventional uniaxial pressing with varying geometry. This has allowed the effects of the multilayer configuration, characterized by a ceramic layer thickness reduced down to tens of microns and an increased number of layers, to be defined. Specifically, a four-time enhancement of magnetoelectric coefficient is obtained, from values of $\sim 7 \mathrm{mV} . \mathrm{cm}^{-1}$. Oe $\mathrm{e}^{-1}$ for the three-layer structures up to $\sim 30 \mathrm{mV} . \mathrm{cm}^{-1}$. Oe $\mathrm{e}^{-1}$ for the multilayer composites. Besides, a distinctive shift of the bias magnetic field for maximum response towards low values take place, so that it is between $1.5-0.85 \mathrm{kOe}$ for the conventionally prepared layered structures and at $\sim 0.5 \mathrm{kOe}$ for the multilayer composites processed by tape casting. Mechanisms behind these effects are discussed, and enhanced magnetoelectric coefficients are associated with the reduction of the piezoelectric single-layer thickness below the characteristic relaxation length of the material system. Relaxation phenomena also controls the position of the field for maximum response, so that multilayering results in unrelaxed 
tensile stressed magnetostrictive layers with high rate of change of magnetostriction with applied magnetic field and thus, composites with decreased bias field for maximum response.

\section{Acknowledgements}

Research co-funded by the Brazilian PVE/CAPES programme (process 88881.030500/2013-01), grants \#2013/00134-7 and \#2017/17872-1 São Paulo Research Foundation (FAPESP), and Spanish MINECO (projects MAT2014-58816-R and MAT2017-88788-R). The authors thank Dr. João Elias Figueiredo Soares Rodrigues by the support in the Raman measurements.

\section{References}

[1] Fiebig M. Revival of the magnetoelectric effect. J Phys D: Appl Phys 2005;38(8):R123. doi:10.1088/0022-3727/38/8/R01.

[2] Eerenstein W, Mathur ND, Scott JF. Multiferroic and magnetoelectric materials. Nature 2006;442(7104):759-765. doi:10.1038/nature05023.

[3] Mandal P, Pitcher MJ, Alaria J, Niu H, Borisov P, Stamenov $\mathrm{P}$, et al. Designing switchable polarization and magnetization at room temperature in an oxide. Nature 2015;525(7569):363-366. doi:10.1038/nature14881.

[4] Fernández-Posada CM, Castro A, Kiat JM, Porcher F, Peña O, Algueró $\mathrm{M}$, et al. A novel perovskite oxide chemically designed to show multi- 
ferroic phase boundary with room-temperature magnetoelectricity. Nat Commun 2016;7:12772. doi:10.1038/ncomms12772.

[5] Vaz CAF, Hoffman J, Ahn CH, Ramesh R. Magnetoelectric coupling effects in multiferroic complex oxide composite structures. Adv Mater 2010;22(26-27):2900-2918. doi:10.1002/adma.200904326.

[6] Hu JM, Chen LQ, Nan CW. Multiferroic heterostructures integrating ferroelectric and magnetic materials. Adv Mater 2015;28(1):15-39. doi:10.1002/adma.201502824.

[7] Bibes M, Barthélémy A. Towards a magnetoelectric memory. Nat Mater 2008;7(6):425-426. doi:10.1038/nmat2189.

[8] Zhao X, Wen J, Yang B, Zhu H, Cao Q, Wang D, et al. Electric field manipulated multilevel magnetic states storage in FePt/(011) PMN-PT heterostructure. ACS Appl Mater Interfaces 2017;9(41):36038-36044. doi:10.1021/acsami.7b11015.

[9] Tsymbal E, Gruverman A, Garcia V, Bibes M, Barthélémy A. Ferroelectric and multiferroic tunnel junctions. MRS Bull 2012;37(02):138-143. doi:10.1557/mrs.2011.358.

[10] Scott JF. Applications of magnetoelectrics. J Mater Chem 2012;22(11):4567. doi:10.1039/c2jm16137k.

[11] Dong G, Zhou Z, Xue X, Zhang Y, Peng B, Guan M, et al. Ferroelectric phase transition induced a large FMR tuning in self-assembled $\mathrm{BaTiO}_{3}: \mathrm{Y}_{3} \mathrm{Fe}_{5} \mathrm{O}_{12}$ multiferroic composites. ACS Appl Mater Interfaces 2017;9(36):30733-30740. doi:10.1021/acsami.7b06876. 
[12] Wang Y, Gray D, Berry D, Gao J, Li M, Li J, et al. An extremely low equivalent magnetic noise magnetoelectric sensor. Adv Mater 2011;23(35):4111-4114. doi:10.1002/adma.201100773.

[13] Wang Y, Li J, Viehland D. Magnetoelectrics for magnetic sensor applications: status, challenges and perspectives. Mater Today 2014;17(6):269275. doi:10.1016/j.mattod.2014.05.004.

[14] Nan CW, Bichurin MI, Dong S, Viehland D, Srinivasan G. Multiferroic magnetoelectric composites: Historical perspective, status, and future directions. J Appl Phys 2008;103(3):031101. doi:10.1063/1.2836410.

[15] Ma J, Hu J, Li Z, Nan CW. Recent progress in multiferroic magnetoelectric composites: from bulk to thin films. Adv Mater 2011;23(9):10621087. doi:10.1002/adma.201003636.

[16] Dong S, Zhai J, Li J, Viehland D. Near-ideal magnetoelectricity in highpermeability magnetostrictive/piezofiber laminates with a (2-1) connectivity. Appl Phys Lett 2006;89(25):252904. doi:10.1063/1.2420772.

[17] Jin J, Zhao F, Han K, Haque MA, Dong L, Wang Q. Multiferroic polymer laminate composites exhibiting high magnetoelectric response induced by hydrogen-bonding interactions. Adv Funct Mater 2013;24(8):1067-1073. doi:10.1002/adfm.201301675.

[18] Martins P, Kolen'ko YV, Rivas J, Lanceros-Mendez S. Tailored magnetic and magnetoelectric responses of polymer-based composites. ACS Appl Mater Interfaces 2015;7(27):15017-15022. doi:10.1021/acsami.5b04102. 
[19] Zheng H, Wang J, Lofland SE, Ma Z, Mohaddes-Ardabili L, Zhao T, et al. Multiferroic $\mathrm{BaTiO}_{3}-\mathrm{CoFe}_{2} \mathrm{O}_{4}$ nanostructures. Science 2004;303(5658):661-663. doi:10.1126/science.1094207.

[20] Algueró M, Ricote J, Torres M, Amorín H, Alberca A, Iglesias-Freire O, et al. Thin film multiferroic nanocomposites by ion implantation. ACS Appl Mater Interfaces 2014;6(3):1909-1915. doi:10.1021/am404945m.

[21] Tan G, Chai Z, Zheng Y, Yue Z, Yang W, Guo M, et al. Tunable structural transition and multiferroic properties of the composite thin films through the structural transition of magnetic layer. J Eur Ceram Soc 2018;38(13):4463-4475. doi:10.1016/j.jeurceramsoc.2018.06.012.

[22] Park CS, Priya S. Cofired magnetoelectric laminate composites. J Am Ceram Soc 2010;94(4):1087-1095. doi:10.1111/j.15512916.2010.04213.x.

[23] Srinivasan G, Rasmussen ET, Gallegos J, Srinivasan R, Bokhan YI, Laletin VM. Magnetoelectric bilayer and multilayer structures of magnetostrictive and piezoelectric oxides. Phys Rev B 2001;64(21). doi:10.1103/physrevb.64.214408.

[24] Islam RA, Ni Y, Khachaturyan AG, Priya S. Giant magnetoelectric effect in sintered multilayered composite structures. J Appl Phys 2008;104(4):044103. doi:10.1063/1.2966597.

[25] Saito Y, Takao H, Tani T, Nonoyama T, Takatori K, Homma T, et al. Lead-free piezoceramics. Nature 2004;432(7013):84-87. doi:10.1038/nature03028. 
[26] Li P, Liu B, Shen B, Zhai J, Zhang Y, Li F, et al. Mechanism of significantly enhanced piezoelectric performance and stability in textured potassium-sodium niobate piezoelectric ceramics. J Eur Ceram Soc 2018;38(1):75-83. doi:10.1016/j.jeurceramsoc.2017.07.017.

[27] Lin Y, Zhang J, Yang H, Wang T. Excellent piezoelectric and magnetoelectric properties of the $\left(K_{0.45} N a_{0.55}\right)_{0.98} L i_{0.02}\left(N b_{0.77} T a_{0.18} S b_{0.05}\right) O_{3} / N i_{0.37} C u_{0.20} Z n_{0.43} F e_{1.92} O_{3.88}$ laminated composites. J Alloys Comp 2017;692:86-94. doi:10.1016/j.jallcom.2016.09.009.

[28] Yang H, Zhang J, Lin Y, Wang T. High curie temperature and enhanced magnetoelectric properties of the laminated $\mathrm{Li}_{0.058}\left(\mathrm{Na}_{0.535} \mathrm{~K}_{0.48}\right)_{0.942} \mathrm{NbO}_{3} / \mathrm{Co}_{0.6} \mathrm{Zn}_{0.4} \mathrm{Fe}_{1.7} \mathrm{Mn}_{0.3} \mathrm{O}_{4}$ composites. Sci Rep 2017;7(1):44855. doi:10.1038/srep44855.

[29] Rosa WS, Venet M, M'Peko JC, Amorín H, Algueró M. Processing issues and their influence in the magnetoelectric performance of ( $\mathrm{K}, \mathrm{Na}) \mathrm{NbO}_{3} / \mathrm{CoFe}_{2} \mathrm{O}_{4}$-based layered composites. J Alloys Comp 2018;744:691-700. doi:10.1016/j.jallcom.2018.02.083.

[30] Brennecka GL, Ihlefeld JF, Maria JP, Tuttle BA, Clem PG. Processing technologies for high-permittivity thin films in capacitor applications. J Am Ceram Soc 2010;93(12):3935-3954. doi:10.1111/j.15512916.2010.04211.x.

[31] Randall CA, Kelnberger A, Yang GY, Eitel RE, Shrout TR. High strain piezoelectric multilayer actuators-a material science and engineer- 
ing challenge. J Electroceram 2005;14(3):177-191. doi:10.1007/s10832005-0956-5.

[32] Amorín H, Algueró M, Campo RD, Vila E, Ramos P, Dollé M, et al. High-sensitivity piezoelectric perovskites for magnetoelectric composites. Sci Technol Adv Mater 2015;16(1):016001. doi:10.1088/14686996/16/1/016001.

[33] Schileo G, Pascual-Gonzalez C, Algueró M, Reaney IM, Postolache P, Mitoseriu L, et al. Multiferroic and magnetoelectric properties of $\mathrm{Pb}_{0.99}\left[\mathrm{Zr}_{0.45} \mathrm{Ti}_{0.47}\left(\mathrm{Ni}_{1 / 3} \mathrm{Sb}_{2 / 3}\right)_{0.08}\right] \mathrm{O}_{3}-\mathrm{CoFe}_{2} \mathrm{O}_{4}$ multilayer composites fabricated by tape casting. J Eur Ceram Soc 2018;38(4):1473-1478. doi:10.1016/j.jeurceramsoc.2017.10.055.

[34] Lin Y, Qiu Y, Zhang J, Yang H, Wang T, Yan F. Transverse and longitudinal magnetoelectric effect in multilayer $\mathrm{Li}_{0.058}\left(\mathrm{Na}_{0.535} \mathrm{~K}_{0.48}\right)_{0.942} \mathrm{NbO}_{3} / \mathrm{Co}_{0.6} \mathrm{Zn}_{0.4} \mathrm{Fe}_{1.7} \mathrm{Mn}_{0.3} \mathrm{O}_{4} \quad$ bulk composites. Appl Phys Lett 2018;112(7):072901. doi:10.1063/1.5008604.

[35] Fu J, Rosa WS, M'Peko JC, Algueró M, Venet M. Magnetoelectric coupling in lead-free piezoelectric $L i_{x}\left(K_{0.5} N a_{0.5}\right)_{1-x} N b_{1-y} T a_{y} O_{3}$ and magnetostrictive $\mathrm{CoFe}_{2} \mathrm{O}_{4}$ laminated composites. Phys Lett A 2016;380(20):1788-1792. doi:10.1016/j.physleta.2016.03.024.

[36] Paulsen JA, Ring AP, Lo CCH, Snyder JE, Jiles DC. Manganesesubstituted cobalt ferrite magnetostrictive materials for magnetic stress sensor applications. J Appl Phys 2005;97(4):044502. doi:10.1063/1.1839633. 
[37] Wang Y, Damjanovic D, Klein N, Hollenstein E, Setter N. Compositional inhomogeneity in Li- and Ta-modified $(\mathrm{K}, \mathrm{Na}) \mathrm{NbO}_{3}$ ceramics. J Am Ceram Soc 2007;90(11):3485-3489. doi:10.1111/j.15512916.2007.01962.x.

[38] $\mathrm{Wu}$ J, Xiao D, Zhu J. Potassium-sodium niobate lead-free piezoelectric materials: Past, present, and future of phase boundaries. Chem Rev 2015;115(7):2559-2595. doi:10.1021/cr5006809.

[39] Algueró M, Calzada ML, Bushby AJ, Reece MJ. Ferroelectric hysteresis loops of $(\mathrm{Pb}, \mathrm{Ca}) \mathrm{TiO}_{3}$ thin films under spherical indentation. Appl Phys Lett 2004;85(11):2023-2025. doi:10.1063/1.1787594.

[40] Eitel RE, Randall CA, Shrout TR, Park SE. Preparation and characterization of high temperature perovskite ferroelectrics in the solidsolution $(1-x) \mathrm{BiScO}_{3}-x \mathrm{PbTiO}_{3}$. Jpn J Appl Phys 2002;41(Part 1, No. 4A):2099-2104. doi:10.1143/jjap.41.2099.

[41] Berganza E, Pascual-González C, Amorín H, Castro A, Algueró M. Point defect engineering of high temperature piezoelectric $\mathrm{BiScO}_{3}-\mathrm{PbTiO}_{3}$ for high power operation. J Eur Ceram Soc 2016;36(16):4039-4048. doi:10.1016/j.jeurceramsoc.2016.07.012.

[42] Pascual-González C, Berganza E, Amorín H, Castro A, Algueró M. Point defect engineering of high temperature piezoelectric $\mathrm{BiScO}_{3}-$ $\mathrm{PbTiO}_{3}$ for enhanced voltage response. Mater Des 2016;108:501-509. doi:10.1016/j.matdes.2016.07.015. 
[43] Amorín H, Ricote J, San-Felipe I, Salazar N, del Campo R, RomagueraBarcelay Y, et al. Multilayer ceramic magnetoelectric composites with tailored interfaces for enhanced response. ACS Appl Mater Interfaces 2017;9(44):39094-39104. doi:10.1021/acsami.7b14775.

[44] Ramos P, Amorín H, Ricote J, Castro A, Algueró M. Insights into the performance of magnetoelectric ceramic layered composites. J Compos Sci 2017;1(2):14. doi:10.3390/jcs1020014.

[45] Pithan C, Shiratori Y, Dornseiffer J, Haegel FH, Magrez A, Waser R. Microemulsion mediated synthesis of nanocrystalline $\left(K_{x}, N a_{1-x}\right) N b O_{3}$ powders. J Cryst Growth 2005;280(1-2):191-200. doi:10.1016/j.jcrysgro.2005.03.038.

[46] Rojac T, Kosec M, Malic B, Holc J. Mechanochemical synthesis of $\mathrm{NaNbO}_{3}, \mathrm{KNbO}_{3}$ and $K_{0.5} \mathrm{Na}_{0.5} \mathrm{NbO}_{3}$. Sci Sinter 2005;37(1):61-67. doi:10.2298/sos0501061r. 